\title{
Advanced Dynamic Spectrum 5G Mobile Networks Employing Licensed Shared Access
}

\author{
G. K. Papageorgiou, K. Voulgaris, K. Ntougias, D. K. Ntaikos, M. M. Butt, C. Galiotto, N. Marchetti, \\ V. Frascolla, H. Annouar, A. Gomes, A. J. Morgado, M. Pesavento, T. Ratnarajah, K. Gopala, \\ F. Kaltenberger, D. T. M. Slock, F. A. Khan and C. B. Papadias
}

\begin{abstract}
Spectrum sharing has been recognized as a key component for $5 \mathrm{G}$ and beyond wireless networks. Recent trials have revealed the business value of spectrum sharing via the Licensed Shared Access (LSA) model, wherein both the incumbent and the licensee operators are protected from harmful interference by sharing the available spectrum using long-term spectrum access agreements. Further increase in capacity gains can be achieved by exploiting Dynamic Spectrum Access (DSA), where access is granted in shorter time intervals, and Spectrum Access System (SAS), which is a more flexible version of traditional LSA. This article first hints at LSA-type spectrum sharing in regulatory and standards bodies, then proposes a few relevant scenarios that would take advantage of LSA and finally presents an LSA system architecture and some enabling technologies for LSA/DSA/SAS. These include novel sensing, dynamic and radioaware resource allocation, and advanced cooperative communication techniques.
\end{abstract}

\section{INTRODUCTION}

$\mathrm{T}$ $\mathrm{HE}$ demand for wireless services and applications is growing faster than ever in recent years. Mobile services are expanding towards data-hungry multimedia services, such as real-time traffic data and $8 \mathrm{~K}$ video. According to a recent forecast, there will be 8.7 billion handheld or personal mobile-ready devices and 4.4 billion M2M connections by 2023 [1]. This tremendous number of terminals will generate a huge volume of data, which is expected to be effectively handled by the $5^{\text {th }}$ Generation (5G) of wireless communication networks. Moreover, an array of future technologies like virtual and augmented reality, holographic projections, and autonomous vehicles will only increase the strain for more capacity on wireless networks.

In view of the above, policy makers have been considering more flexible licensing approaches that go beyond rigid spectrum allocation and promote spectrum sharing between operators and services. New concepts for spectrum management have been thus proposed and are being pushed into standards, most notably Licensed Shared Access (LSA), Dynamic Spectrum Access (DSA) and Spectrum Access System (SAS). This article provides an overview of such flexible spectrum sharing approaches and reports a number of relevant advances that were obtained under several EU-funded collaborative research projects [2]-[5].

This article is organized as follows. Section II focuses on the LSA/DSA/SAS (LSA-type) regulatory and standards aspects. Section III defines some key LSA-type scenarios. Section IV proposes an innovative LSA architecture. Section V details some novel proposed technology enablers. Section VI elaborates on the experimentations and the achieved results. Finally, Section VII concludes the article.

\section{REGULATORY AND STANDARDIZATION ASPECTS}

\section{A. Regulatory bodies}

Regulation of spectrum sharing started about two decades ago with cognitive radio-type solutions in unlicensed bands and TV white spaces. As such regulation could not ensure any quality of service (QoS) to licensed (and much less so to unlicensed) users, it did not attract much interest from telecom operators, however it gave way to the so-called LSA spectrum sharing paradigm. The Radio Spectrum Policy Group (RSPG), which assists the European Commission (EC) in the development of radio spectrum policy, started in 2013 to publish its positive opinion on LSA, and since then fosters its adoption in commercial networks, stressing how LSA might achieve more spectrum efficiency and create new use cases with high market potential [6]. Thanks also to that input, the EC directed the European Conference of Postal and Telecommunications administrations (CEPT), another European coordination body, to review spectrum sharing opportunities with the aim to harmonize LSA deployment across the EU. The CEPT thus recommended the 2.3-2.4 GHz bands for initial LSA deployment. In most EU countries, these bands are allocated for use by Program Making \& Special Events (PMSE) operators [4].

Outside Europe, beyond traditional LSA, the US Federal Communications Commission (FCC), and the Australian Communications and Media Authority (ACMA) proposed the use of a similar concept to LSA, i.e. the Dynamic Spectrum Access (DSA) [3] in bands used by radars. The FCC proposed a threetiered framework, wherein federal, priority access and general access operators are granted a varying priority to spectrum access. In addition, a network of dedicated sensors detects radar activity and notify the priority and general access operators, maximizing the spectrum sharing opportunities, while protecting the federal operators from harmful interference. Moreover, the FCC has introduced the Spectrum Access System (SAS) concept, mainly in relation to Citizens Broadband Radio Service (CBRS) networks [7]. The SAS is based on LSA but allows for more flexibility and ensures coexistence with incumbent users, who are not able to provide any a priori information to a central access database.

\section{B. Standards bodies}

The ongoing LSA and SAS standardization process follows the CEPT recommendations, focusing on the $2.3 \mathrm{GHz}$ band, considered as additional spectrum for mobile operators. The main standards bodies involved in LSA are the Workgroup 1 of the Technical Committee on Reconfigurable Radio Systems of the European Standardization Telecommunication Institute (ETSI TC RRS WG1), and the Workgroup 5 of the Technical Specification Group on Services and Systems Aspects of the 3rd Generation Partnership Project (3GPP TSG SA WG5), with inputs coming 


\begin{tabular}{lccc}
\hline & $\begin{array}{c}\text { Scenario I } \\
\text { Railway }\end{array}$ & $\begin{array}{c}\text { Scenario II } \\
\text { Macro-Cell }\end{array}$ & $\begin{array}{c}\text { Scenario III } \\
\text { Small-Cell }\end{array}$ \\
\hline $\begin{array}{l}\text { Suggested } \\
\text { LSA band }\end{array}$ & 2300-2400 MHz & $2300-2400 \mathrm{MHz}$ & $\begin{array}{c}2300-2400 \mathrm{MHz} \\
3500-3600 \mathrm{MHz}\end{array}$ \\
\hline $\begin{array}{l}\text { LSA } \\
\text { Application }\end{array}$ & $\begin{array}{c}\text { Backhaul link inside } \\
\text { moving trains for mobile } \\
\text { broadband connectivity }\end{array}$ & $\begin{array}{c}\text { Extra capacity for any } \\
\text { service deployed by the } \\
\text { operator }\end{array}$ & $\begin{array}{c}\text { Extra capacity for } \\
\text { mobile broadband }\end{array}$ \\
\hline Incumbent & $\begin{array}{c}\text { Aeronautical telemetry } \\
\text { stations and military } \\
\text { stations }\end{array}$ & $\begin{array}{c}\text { Aeronautical telemetry } \\
\text { stations and military } \\
\text { stations }\end{array}$ & $\begin{array}{c}\text { Wireless cameras, } \\
\text { Fixed wireless } \\
\text { broadband }\end{array}$ \\
Licensee & Railway operator & $\begin{array}{c}\text { Mobile network } \\
\text { operators }\end{array}$ & $\begin{array}{c}\text { Mobile network } \\
\text { operators }\end{array}$ \\
\hline
\end{tabular}

Table 1: LSA Reference scenarios and their corresponding parameters [5]

from the CBRS Alliance.

The ETSI, in its first version of the LSA standard, proposed that mobile operators access the available LSA band according to static or semi-static predefined frequency plan(s) and to the proposed system reference parameters. This approach, while somewhat conservative, fosters a fast and simple implementation to facilitate an initial LSA adoption. According to the ETSI standard, every mobile operator should deploy an LSA Controller that would connect to an LSA Repository (LR) to check that the "preallocated' channels are not in use by the corresponding incumbent. More recently, ETSI completed the definition of the LSA requirements, the specification of the system architecture and related network procedures, and the protocol details for the implementation of these procedures.

In 3GPP some preliminary discussions started already in the 3GPP Rel-14 timeframe. However, it is with the recently concluded Rel-15, and even more with the forthcoming Rel-17 (which, according to the latest 3GPP SA plenary meeting, held in March 2020, will be completed in September 2021) that effective specifications are expected to be published.

Finally, the CBRS Alliance aligned its Release 3 specifications with the content of 3GPP Rel-15, and is currently working on Release 4, which will match the work ongoing in 3GPP Rel-16/17.

\section{EXEMPLARY LSA SCENARIOS}

The application of LSA may vary from extremely short to significantly long allocation periods, from short range to wider service areas, and may address varying bandwidth requirements, as outlined in three exemplary reference scenarios, proposed by the EU-funded project ADEL [5], characterized in Table 1, and briefly described below.

Scenario I "Railway": The LSA spectrum is added to the pool of bands currently available in order to support additional backhaul links between trains in motion and static base stations placed along the tracks. The LSA licensee, e.g., the railway operator, must request in advance access to spectrum in the LSA band (assumed 2.3-2.4 GHz) in the specific portion of the track that the train will pass through, according to a specific timetable [5].

Scenario II "Macro-Cell": In this scenario, LSA resources are used to support links between base stations and end-user devices in macro cells. These LSA resources are used as added spectrum to provide more capacity to macro cellular networks in a costefficient manner. The LSA resources are shared in time and space with the incumbents that exist in the coverage range of mobile network operators (MNO). There must be a prior agreement between the LSA licensee and the LSA system regarding the use of the LSA resources, including the time slots that the resources are available for use by the MNO [5].

Scenario III "Small-cell": The LSA resources are deployed in small-cells in densely populated areas to provide added capacity for mobile broadband services at reduced cost. The deployment of LSA small cells in this scenario is integrated with legacy micro/macro-cells. Due to anticipated high demand for spectrum in such areas, two LSA bands are to be used for the small cells, i.e. 2.32.4 GHz and 3.5-3.6 GHz. Both LSA bands need to be shared with the incumbents, e.g., wireless cameras in the $2.3 \mathrm{GHz}$ band or rural fixed broadband operators in the $3.5 \mathrm{GHz}$ band [5].

\section{Proposed LSA ARChiteCtURE}

The proposed system architecture facilitates the implementation of the LSA vision as defined by the CEPT, which consider LSA as a spectrum sharing approach implicitly associated with dynamic and coordinated radio resource management [5]. Such architecture, shown in Fig. 1, is based on an "LSA Band Manager" (LBM) that coordinates the access of several incumbent and licensee operators to the LSA band, according to agreed sharing rules. It includes an LR that contains the information of spectrum utilization by the incumbent(s), and acts as a proxy between them and the LBM, hiding confidential information, when required.

The frequency allocation decisions, made well in advance by the LBM, are assisted by a radio propagation database called "Radio Environment Map" (REM), intended to keep updated information about the radio environment conditions along time and space. The information on this database results from four sources: i) the LR, which contains availability information provided by the incumbent operators; ii) the LBM, that provides information on resource allocations that have been scheduled; iii) internal propagation calculations that behave as initial estimations of the impact of these allocations on the radio environment; and, iv) information from several spectrum sensing networks consolidated by the spectrum sensing reasoning module. It should be noted that spectrum sensing information in LSA architecture is utilized in following ways: a) to make fine adjustments in the propagation models within the REM; b) to gather information not available from the incumbents through the LR; c) to detect sharing agreement violations; and, d) to collect band usage statistics that may be made available to the LBM to improve future frequency allocation decisions [5].

As mentioned earlier, the FCC has recently introduced SAS, which considers additional flexibility with respect to LSA. A key challenge for standards bodies will be how to best combine both approaches, i.e., LSA for Europe and SAS for the US, into a single 


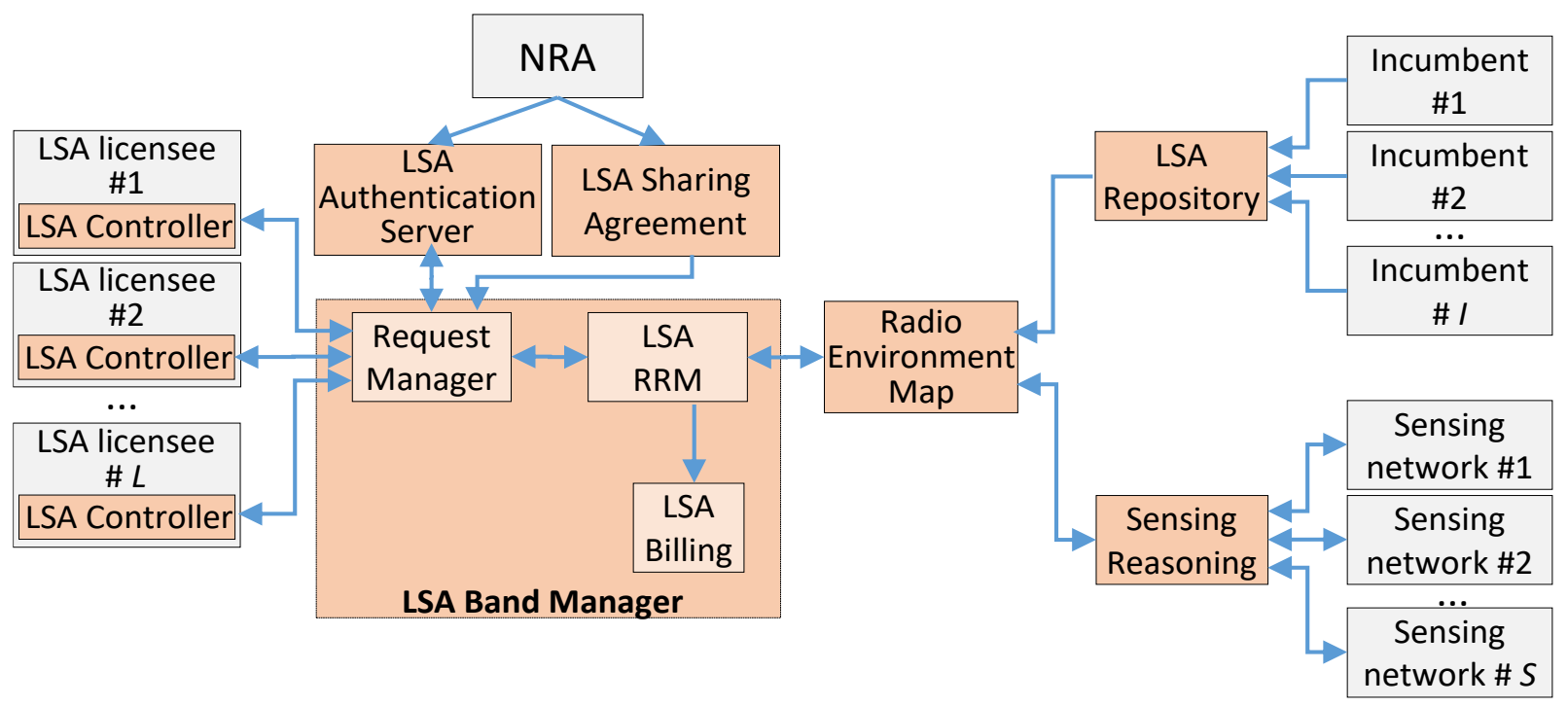

Figure 1: A proposed LSA system architecture

integrated framework. Thanks to its generic approach, the proposed LSA architecture can be considered as a unified framework for implementing different spectrum sharing approaches. The proposed architecture was the base for some documents (RRSWG1(17)040011 and RRSWG1(17)040012), presented and discussed at ETSI RRS WG1 meeting, held at Mainz, Germany in November 2017. That discussion started a stream of activities that are still ongoing, aiming at adding more dynamicity in time, to the way spectrum can be used in $5 \mathrm{G}$ and beyond deployments.

\section{NEW ENABLING TECHNOLOGIES}

Our ambition is to prove that LSA can be the $5 \mathrm{G}$ mobile broadband enabler, which makes it possible to benefit from higher spectral efficiency offered by spectrum sharing. To achieve this vision, we promote fundamental research in three constituent enabling technologies: a) collaborative sensing; b) radio-aware resource allocation; and, c) cooperative communication. The rest of this section presents the major scientific achievements in the mentioned relevant fields.

\section{A. Collaborative Sensing}

Collaborative sensing is a key enabling technology for dynamic spectrum sharing in LSA. In order to reliably identify available spectral resources and shared access opportunities, efficient spectrum sensing algorithms are of fundamental importance. Collaborative sensing, where multiple sensing devices exchange sensing information in a centralized or in a decentralized architecture, is known as a powerful remedy to overcome radio sensing problems, such as the hidden node problem that occurs when an active node is outside the coverage area of the sensing node. Collaborative spectrum sensing for opportunistic authorized access needs to deal with several trade-offs that should be addressed to enable coexistence of the incumbent and licensee networks.

Sensing time vs. communication time: In high mobility scenarios, such as Scenario II, ultra-fast decisions on the availability of spectral resources are required to allow secondary transmissions, increasing the overall spectral efficiency. However, fast sensing can lead to errors and unintended disturbance of the incumbent network, due to false detection of spectral holes.

Collaboration vs. communication overhead: Collaboration among the sensing nodes enhances the reliability of spectrum sensing by exploiting the spatial dimension. The degree of collaboration in the sensing network and the resulting overall spectral efficiency of the LSA network is however fundamentally limited by the associated communication overhead, which is required for collaboration.

Prior information vs. generic sensing: Collaborative sensing can greatly benefit from the use of prior information regarding the incumbent network. Prior knowledge can exist in form of specific known signal characteristics, such as frame and reference structures, knowledge of the geographic location of the incumbent network transceivers, and/or the channel characteristics. This information can be exploited by the sensing process to enhance the overall network performance.

All the technical challenges mentioned above are addressed with the development of novel distributed sensing schemes that use three distinctive features to enhance the sensitivity and reliability of the sensing: i) the massive cooperation between sensing devices; ii) the exploration of the spatial domain through directional multi-antenna processing; and, iii) the incorporation of contextual-side information using database aided sensing technologies.

In this context, compressed sensing (CS) and sparse modelling techniques, that rely on parametric measurement models characterized by only a few parameters, have been identified as particularly useful for this application, due to their ability to produce high resolution multidimensional spectral and spatial sensing information from low sample size measurements. Therefore, we developed a novel optimization framework based on successive convex function approximation, with application in CS that can be efficiently implemented on highly parallel modern hardware architectures [8].

In the case that the radio access technology, e.g., the precise frame, signal or reference structures, used by the incumbent network is known, this information can be exploited to further enhance the sensing performance. Such prior information can be used in a preprocessing step to simplify the sensing procedure. Interference from competing LSA network that coexists with the incumbent network may e.g., be suppressed based on a known reference signal structure with the objective to enhance the signal quality and to reduce the data dimension of the sensing problem.

We propose a dedicated "Spectrum Sensing Reasoning" module that uses the spectral and spatial sensing information obtained from the collaborative sensing algorithms to maintain dedicated sensing maps, similar to a standard radio coverage map. However, 


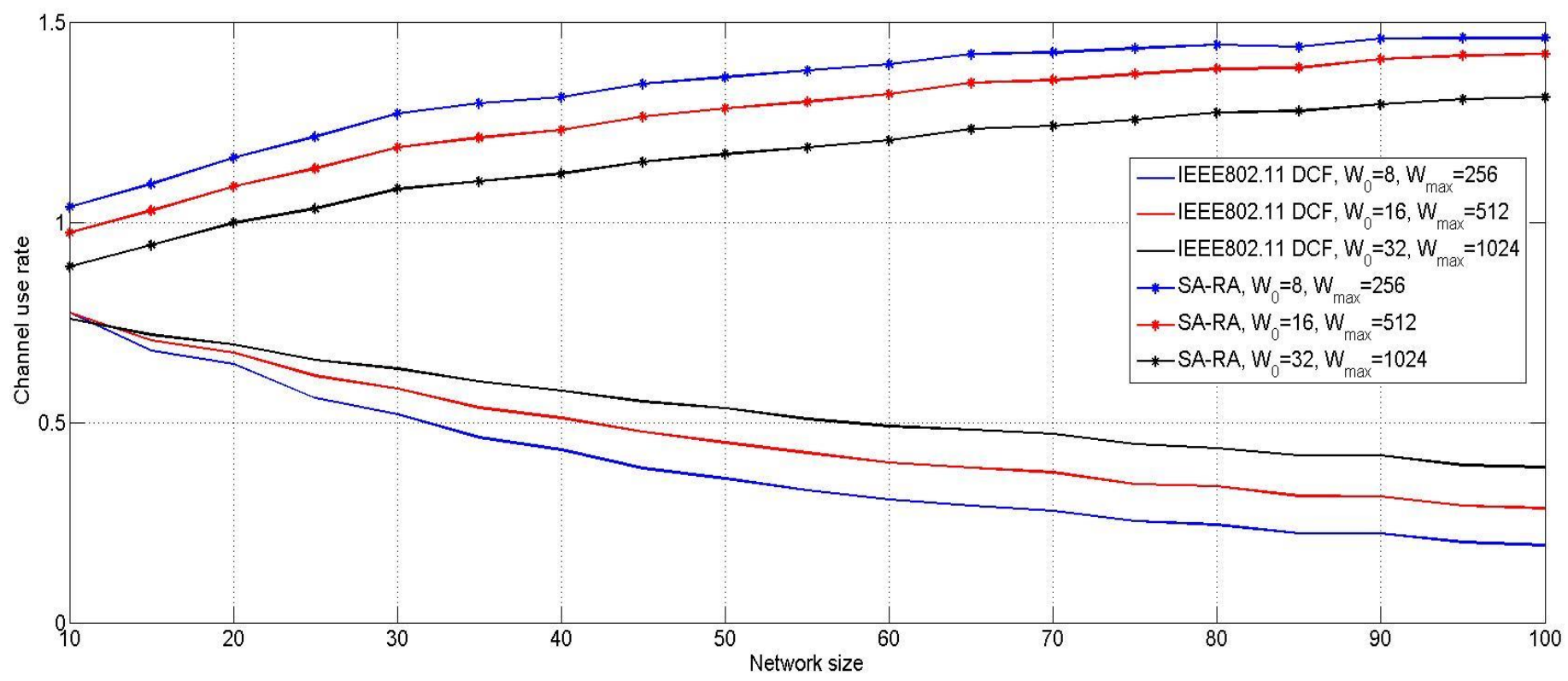

Figure 2: Channel user rate vs. network size for IEEE802.11 DCF and SA-RA.

this module also controls several sensing networks and control functions, including the detection of faulty measurements and the request of additional measurements for specific zones. The information obtained by this module, together with incumbentspecific information, forms the radio resource map that is used by the central LSA controller.

\section{B. Radio-aware Resource Allocation}

\section{1) Random Access Protocols}

In standard frequency allocation schemes, different cells may use the same channel only if all User Equipment (UE) of each cell do not interfere with any UE of the other ones, hence reducing the spatial reuse possibilities of the spectrum. Moreover, sharing all available LSA bands is more efficient than dedicating an LSA band to each cell, since it is more robust against fluctuation of their availability and against changes in the network topology. A distributed channel access scheme, on UE level, is then preferable as per-node based frequency reuse is less constrained than per-cell based frequency reuse. This solution is also in line with the objective of having Device to Device (D2D) communications capability, on dedicated or shared spectrum, in the $5 \mathrm{G}$ standards.

The IEEE802.11 Distributed Coordination Function (DCF) protocol is the most popular distributed access scheme. It operates on single channel, performs well in single-hop topologies, but suffers from severe performance degradation in multi-hop ones. The new distributed Medium Access Control (MAC) protocol introduced in [9], called Synchronized Acknowledgment Random Access MAC (SARA MAC), solves efficiently, in its single channel version, the hidden node problem without introducing the masked node problem.

In its multichannel version, the protocol also solves the exposed node problem and allows a complete channel reuse. The protocol operates on synchronous networks and defines dedicated slots for Clear To Send (CTS) messages in a frame basis. Communications may be established at any slot of the frame, but they last, at maximum, until the end of the frame. Hence, preventing nodes that detect transmission on CTS slots from accessing the channel until the end of the frame is enough for resolving the hidden node problem even in masked situations. In Fig. 2, much superior performance of the new distributed MAC protocol in comparison with the IEEE802.11 DCF protocol for different network sizes in multi-hop topologies is demonstrated [9].

\section{2) Spectrum Allocation}

In most of the LSA literature, spectrum allocation is based on some auction mechanism, where the spectrum is allocated (when available) to the MNOs on a long term-basis. This has the drawback that the spectrum is wasted if the operator owning the spectrum does not require it due to low network demand at a particular instant. In [10], we have assumed that at the time of spectrum allocation, no formal bidding process is considered and that the MNOs consent in advance on a fair use of shared spectrum resources such that each MNO pays the same price and receives a fair share of the accessible LSA spectrum. The proposed spectrum allocation algorithm operates in a proportionally fair manner and assigns spectrum to the operators that have spectrum demand, based on their allocation history.

It is shown in [10] that the proposed algorithm provides fair spectrum allocation to all the MNOs, regardless of their demand, i.e., spectrum hungry MNOs do not get more spectrum in the long run as all the MNOs have paid the same price. It is worth noting that the proposed algorithm promises to meet the temporal spectrum demand of the MNO when it is scheduled to access spectrum, but its priority index will be large as a result, and the corresponding probability to get spectrum access in next time slot will decrease, following the principle of proportional fairness. Resource allocation schemes that consider user misbehavior and penalization are also developed in [11, [15].

\section{Cooperative Communication}

The operation of LSA systems must be designed in a way that QoS, in the form of spectral, energy, and cost efficiency, is guaranteed for both incumbent and licensee systems, while accessing the shared spectrum. However, in order to achieve a joint, requested QoS objective, cooperation needs to characterize the operation between licensee operators/devices, as well as the concurrent transmissions of incumbent and licensee systems.

We have developed, in reference to the investigated scenarios, several algorithms and schemes that facilitate cooperative communication between entities belonging to an LSA network. Focusing on the small-cell scenario (Scenario III), the case where 

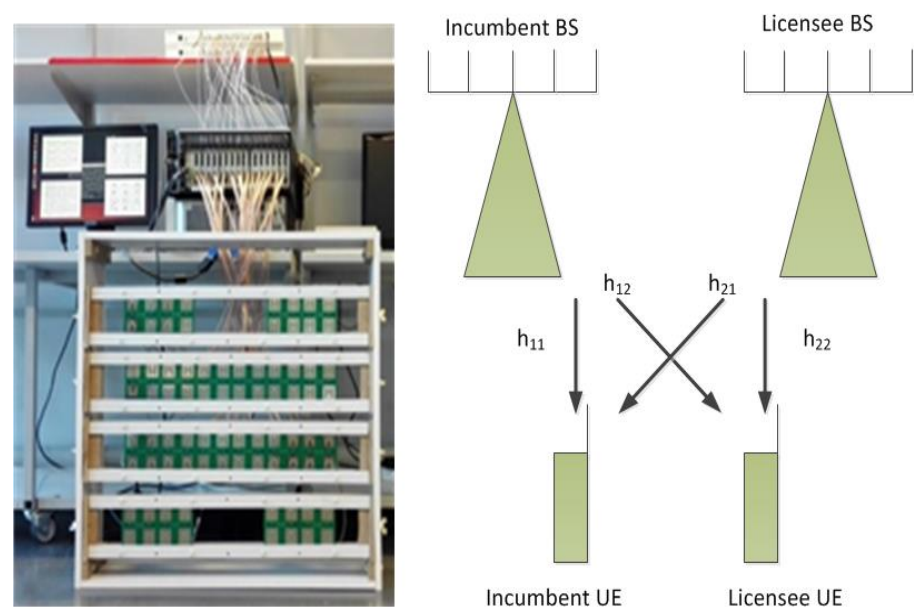

Figure 3: Demo Test bed and scenario for Spatial LSA.

several users require wireless service from a Virtual Mobile Network Operator (VMNO), is studied. Motivated by the above situation, a cost efficiency metric is proposed [12], and then, based on that and on the Zero-Forcing (ZF) distributed Multiple-InputMultiple-Output (MIMO) precoding technique, the optimal solution is evaluated in terms of cost efficiency, in comparison to an arbitrary, uncoordinated strategy. It is interestingly shown that the optimal scheme achieves a gain in cost efficiency, for several system scenarios.

Furthermore, a multi-antenna spatial coexistence scenario is considered, in which beamforming (BF) allows to control the mutual interference. The Time Division Duplex (TDD) mode, which is coincidentally the main mode envisaged for Massive MIMO and cellular 5G, largely facilitates this coexistence due to the possible exploitation of channel reciprocity [13]. LSA cooperation allows synchronization, estimation of direct and cross link channels, and hence implements optimal coordinated BF (such as Zero-Forcing (ZF) at high SNR). We have also explored intermediate solutions, e.g., with instantaneous Channel State Information at the Transmitter (CSIT) for the intra-system links, but only covariance CSIT for the inter-system links.

\section{EXPERIMENTATION AND SimULATIONS}

Our contribution to the usage of LSA goes beyond conservative ways of using the spectrum. We show that spectrum can be utilized more efficiently and without a compromise in performance guarantees. This section is devoted to the presentation of results of over-the-air experimentations based on some of the abovementioned enabling technologies.

\section{1) Collaborative Sensing}

The goal is to validate that collaborative spectrum sensing techniques can enable spectrum sharing between incumbent and licensee operators in a more dynamic way, thus increasing the spectral efficiency. We aim at identifying spectrum holes within the shared frequency band for better utilization of the spectrum.

Our setup includes an incumbent link (transmitter and UE), licensee UEs (LU) distributed in the entire area and a sensing network. The sensing network consists of two sensing nodes. Each node comprises six printed parasitic antenna arrays. Each antenna is separated by thin copper plate for extra sectorial isolation. Both nodes were connected to a National Instruments' Universal Software Radio Peripheral (USRP). The incumbent transmitter (Tx) transmits OFDM symbols at a bandwidth of $5 \mathrm{MHz}$ over 52 subcarriers (out of 64-split in 4 channels of 13 subcarriers). The objective is to detect its activity in the spectral and spatial domain by applying efficient spectrum sensing techniques. Based on the channel occupancy, transmission is performed by the licensees in idle channels or in sectors that do not interfere with the incumbent.

The sensing network collects data and processes them according to one of the following collaborative schemes: i) each observation (from each one of the twelve sectors) is processed separately, ii) observations are combined according to a distributed scheme that sums the energy of sectors pointing at the same direction. Finally, an energy detector performs the classification of the channel (idle vs. occupied), based on the OR rule. It is observed that the proposed collaborative (distributed) approach reduces misdetection occurrences significantly.

\section{2) Spatial LSA Proof-of-Concept}

The spatial LSA shows that multiple antennas can permit incumbent and licensee systems to coexist simultaneously, thanks to coordinated BF. Fig. 3 shows an image of the Massive MIMO demo setup and the demo scenario. The Massive MIMO array is built with 12 microstrip antenna cards for practical validation. Each microstrip antenna card has 4 antennas, leading to 48 overall antennas. These antennas are steered by 12 ExpressMIMO2 radio cards with each radio card having 4 transceivers. The design of beamformer is applied on every frequency subcarrier individually for 5MHz LTE transmission which uses 300 occupied subcarriers [14].

The proof of concept also shows that various levels of LSA cooperation are possible with respect to knowledge of the crosslink channels. In particular, semi-blind techniques, which require only intra-cell channel estimation, have shown great potential. The performance of ZF, which needs complete knowledge of direct and cross-link channels, is compared with that of semi-blind techniques that only use statistical information for the cross-link. It is shown that with careful design, even semi-blind techniques can perform close to non-blind techniques. The experiments have also shown that independent and identically distributed (i.i.d) channel models may not be applicable and that the resulting channel hardening may depend on the environment. Nevertheless, Massive MIMO with appropriate transmission techniques shows great potential for substantial increase in throughput and other performance indicators.

\section{3) System-Level Simulation and Misbehavior Detection}

We propose a System-Level Simulator (SLS) for LSA systems, i.e., a simulation platform that implements the key components of an LSA network, such as, the incumbents, the LBM and repository, several MNOs, as well as a Dedicated Sensing Network (DSN). The implemented framework in the SLS, comprises of a) misbehavior detection performed by means of a DSN, b) penalization of misbehaving MNOs, and c) penaltydriven resource allocation [15). We have shown that this proposed framework enhances the compliance of MNOs to the LSA sharing policy, as it provides them an incentive not to misbehave.

Finally, we have also investigated the Area Spectral Efficiency (ASE) gain of the proposed LSA architecture, which we define as the ratio between the ASE of the LSA system (including incumbents and MNOs) and that of a standard non-LSA system, where only the incumbents operate. This gain is obtained due to an enhanced exploitation of the spectrum over space, which is achieved by activating macro-cells on a per-sector basis, in a way that guarantees the incumbents a targeted Signal-to-Interferenceplus-Noise-Ratio (SINR). Depending on the activity of the incumbent, we observe that the LSA gain in terms of ASE ranges from an order of 10 to an order of 100 as shown in Fig. 4. The 


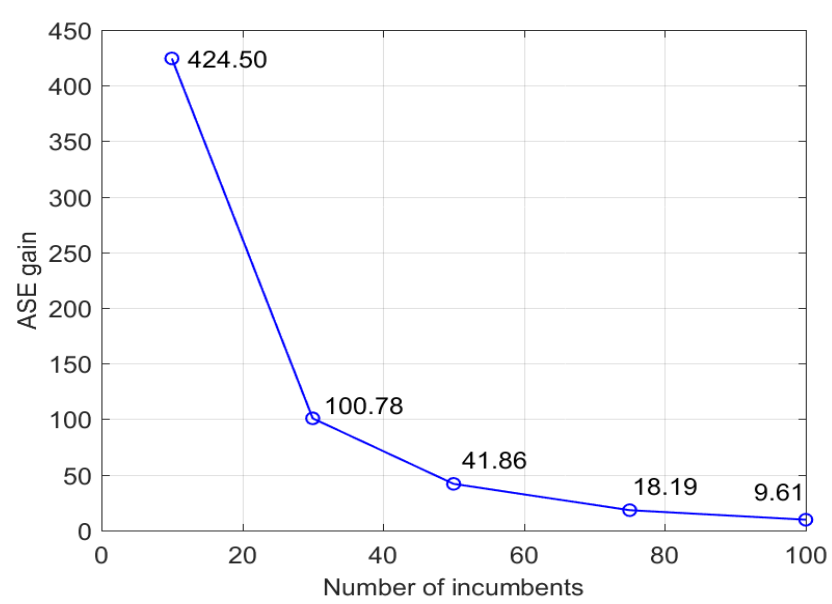

Figure 4: ASE gain of the LSA system as a function of the number of incumbents over an area of $4.33 \mathrm{Km} \times 5 \mathrm{Km}$. Incumbents transmit power is $20 \mathrm{dBm}$, while duty-cycle is $10 \%$.

ASA gain is higher when the number of incumbents is small, due to the more efficient usage of the available spectrum, which is allowed by the introduction of the LSA system.

\section{CONCLUSIONS}

In this article, we provide a survey of some key enabling technologies for an effective deployment of upcoming spectrum sharing setups, i.e., LSA, DSA and SAS. We start by hinting at regulatory and standardization aspects, then we introduce some exemplary scenarios that would benefit from the introduction of LSA systems. Finally, we summarize a few novel results in sensing, dynamic and radio-aware resource allocation, and advanced cooperative communication techniques that were developed to further the potential of LSA-type spectrum sharing for future wireless networks, i.e., 5G and beyond systems.

\section{ACKNOWLEDGEMENT}

This work has been supported by the European Commission FP7 Research Project ADEL (Grant Agreement No: 619647).

\section{REFERENCES}

[1] Cisco, "Cisco Annual Internet Report (2018-2023) White Paper", [Online] Available: https://www.cisco.com/c/en/us/solutions/collateral/executiveperspectives/annual-internet-report/white-paper-c11-741490.html, Accessed on Apr. 27, 2020.

[2] S. Mumtaz et al., "Dynamic Spectrum Management for 5G", IEEE Wireless Communications, 24 (5), 12-13, 2017.

[3] U. Herzog et al., "Quality of Service Provision and Capacity Expansion Through Extended-DSA for 5G," European Conference on Networks and Communications (EuCNC), Athens, Greece, June 27-30, 2016.

[4] Guirao MDP et al., "Locally and Temporary Shared Spectrum as Opportunity for Vertical Sectors in 5G", IEEE Network, vol. 31(6), pp. 24-31, Nov 2017.

[5] C. B. Papadias, T. Ratnarajah, D. T. M. Slock (Eds.), "Spectrum Sharing: The Next Frontier in Wireless Networks" Wiley, Apr. 2020.

[6] RSPG, "Work programme for 2020 and beyond," RSPG19-029, Oct, 2019.

[7] CBRS Alliance, "CBRS: Should the enterprise and venue owners care?,", White Paper, May 2019, [Online] Available:https://senzafili.com/wpcontent/uploads/2019/01/SenzaFili_CBRS_DeepDiveReport.pdf, Accessed on Apr. 27, 2020.

[8] Y. Yang and M. Pesavento, "A Unified Successive Pseudoconvex Approximation Framework," IEEE Trans. Signal Process., vol. 65 (13), pp. 3313-3328, Jul. 2017.

[9] H. Anouar, "Access Protocol and Method in an Ad Hoc Network", FR3031271/ EP3041309/ US2016192359, 2014/12/30.
[10] M. M. Butt, et al., "Fair Dynamic Spectrum Access in Licensed Shared Access Systems", IEEE Int. Symp. Personal, Indoor and Mobile Radio Commun., Valencia, Spain, Sep. 2016.

[11] M. M. Butt et al., "Fair Dynamic Spectrum Management in Licensed Shared Access Systems," IEEE Sys. Journal, vol. 13, no. 3, pp. 2363-2374, Sept. 2019.

[12] M. C. Filippou et al., "Coordinated Shared Spectrum Precoding With Distributed CSIT," IEEE Trans. Wirel. Commun., vol. 15(8), pp. 5182-5192, Aug. 2016.

[13] X. Jiang et al., "A Framework for Over-the-air Reciprocity Calibration for TDD Massive MIMO Systems," IEEE Trans.Wireless Commun., vol. 17(9), Sept. 2018, pp. 5975-5990.

[14] K. Gopala and D. Slock, "Robust LMMSE Beamformer Design by Naive UL/DL Duality and Validation for Non-Cooperative Massive MIMO," IEEE 88th Veh. Tech. Conference, Chicago, USA, 2018.

[15] C. Galiotto et al., "Unlocking the Deployment of Spectrum Sharing with a Policy Enforcement Framework," IEEE Access, vol. 6, pp. 11793-11803, 2018.

\section{BIOGRAPHIES}

Georgios Papageorgiou is currently a Research Associate at Heriot-Watt University, Edinburgh. His research activities include machine learning, signal processing with applications to wireless networks, compressive sensing and adaptive filtering, mathematical modeling and optimization.

Konstantinos Voulgaris is a Smart Development Adviser at JASPERS / EIB assisting the preparation of major infrastructure projects across EU member states. He has over 15 years of experience across engineering, research, and regulatory roles in Motorola, T-mobile, Bell Labs, Ofcom, and AIT.

Konstantinos Ntougias is a Research Associate at the University of Cyprus. His research interests include MIMO communications, resource allocation, spectrum sharing, wireless power transfer, and caching.

Dimitrio Ntaikos is a Senior Research Scientist at the B-WiSE Lab of AIT, Greece focusing in novel antenna designs. He has worked on various externally funded European projects, as well as industry-funded research projects over the past decade.

Majid Butt is Senior Research Specialist 5G+ at Nokia Bell Labs, France. His research interests include communication techniques for wireless networks, focusing mainly on radio resource allocation, scheduling algorithms, energy harvesting and green communication techniques.

Carlo Galiotto is currently working as research engineer at Aspire Technology, Dublin, Ireland. His current research interests include the study of performance and issues of extremely dense networks and small cells, and spectrum sharing for 5G systems.

Nicola Marchetti is an Associate Professor in Wireless Communications at Trinity College Dublin, Ireland. His research interests include radio resource management, self-organising networks, complex systems science and signal processing for communication networks.

Valerio Frascolla is Director of research and innovation at Intel, Germany. He has expertise on $5 \mathrm{G}$ systems business, exploitation, and standardization matters. His research interest is on $5 \mathrm{G}$ system design, with focus on machine learning algorithms, spectrum management, mmWaves, and MEC technologies.

Hicham Anouar is Founder \& CTO of Phanteq, a company providing innovative patented distributed access solutions for wireless communications networks. Earlier, he worked as radio assess architect at Thales Communications, France.

Álvaro Gomes is currently working in Altice Labs - Innovation and Technology Strategy group as technical consultant on mobile 
networks. He participated in several research projects co-funded by the European Commission.

Antonio Morgado is currently with the Mobile Systems Group at the Instituto de Telecomunicacoes, Portugal. His current research interests include spectrum regulation, $5 \mathrm{G}$ standardization, radio resource management, D2D communications, and mmWave communications.

Marius Pesavento is a Professor in communication systems at Technische Universität Darmstadt, Germany. His research interests include robust signal processing and adaptive beamforming, statistical signal processing, spectral analysis, and parameter estimation.

Tharmalingam Ratnarajah is a Professor in digital communications and signal processing at the University of Edinburgh, UK. His research interests include 5G and beyond wireless networks, full-duplex radio, mmWave communications.

Kalyana Gopala obtained the Ph.D. degree from Eurecom, France. His research interests are in wireless communication systems and signal processing for communications. He currently works as Technical Lead at Sequans Communications, Sophia Antipolis, France.

Florian Kaltenberger is an Assistant Professor at the Communication Systems Department of Eurecom, SophiaAntipolis, France. His research interests include 5G, software defined radio, and signal processing for wireless communications.

Dirk Slock is a Professor with Department of Communication Systems, Eurecom, Sophia-Antipolis, France. His research interests include statistical signal processing and signal processing techniques for wireless communications. He is a Fellow of IEEE and EURASIP.

Faheem A. Khan is a Senior Lecturer at School of Computing and Engineering, University of Huddersfield, UK. His research interests include cognitive radios, spectrum sharing, nonorthogonal multiple access, and full-duplex communications.

Constantinos B. Papadias is the Executive Director of the Research, Technology and Innovation Network (RTIN) at The American College of Greece, where he is also Professor of Information Technology. He is also Adjunct Professor at Aalborg University and at the University of Cyprus. He is a Fellow of IEEE and of the European Alliance of Innovation. 